BULL. AUSTRAL. MATH. SOC.

\title{
GRAPHS WITH EULERIAN CHAINS
}

\author{
Roger B. Eggleton and Donald K. Skilton
}

An eulerican chain in a graph is a continuous route which traces every edge exactly once. It may be finite or infinite, and may have 0,1 or 2 end vertices. For each kind of eulerian chain, there is a characterization of the graphs which admit such a tracing. This paper derives a uniform characterization of graphs with an eulerian chain, regardless of the kind of chain. Relationships between the edge complements of various kinds of finite subgraphs are also investigated, and hence a sharpened version of the eulerian chain characterization is derived.

\section{Introduction}

An eulerian chain in a graph is a continuous route which traces every edge of the graph exactly once. Eulerian chains are of four possible types :

(1) eulerian trail, a finite eulerian chain with two end vertices;

(2) eulerian circuit, a finite eulerian chain with no end vertices;

(3) eulerian one-way chain, an infinite eulerian chain with exactly one end vertex;

Received 19 December 1983.

Copyright Clearance Centre, Inc. Serial-fee code: 0004-9727/84 $\$ \mathrm{~A} 2.00+0.00$. 
(4) eulerian two-way chain, an infinite eulerian chain with no end vertex.

A characterization of the graphs which have an eulerian circuit is wellknown; so too is a characterization of those graphs which have an eulerian trail. These results are due to Euler [3], hence the present terminology. Less widely known are characterizations of the graphs which have an eulerian one-way chain, and those which have an eulerian two-way chain. The problem of characterizing such graphs was solved by Erdös, Grünwald (= Gallai) and Vázsonyi [2]. For convenience all these results are stated in Section 3. The purpose of the present paper is to synthesize these results into a single characterization of the graphs which have an eulerian chain, without explicit separation into cases dependent on the nature of that chain. In giving such a characterization, we shall be able to place certain additional restrictions on the conditions given by [2].

The following terminology is convenient. An even graph is any graph with no vertex of odd degree (though vertices of infinite degree are not excluded). An eulepian graph is a connected finite even graph. A quasieuzerian graph is a connected countably infinite even graph. (Some authors omit connectedness from their definitions of the last two terms, and allow quasi-eulerian graphs to be uncountably infinite.)

If $H$ is a subgraph of a Eraph $G$, the erge complement of $H$ in $G$ is the graph $G \backslash E H$ induced by the edges of $G$ which are not edges of $H$. The cofinite rank of $G$ is the smallest cardinal $\alpha$ such that for every finite subgraph $H$ of $G$, the number of infinite components in $G \backslash E H$ is a.t most $\alpha$. We similarly define the connected cnfinite rank, the even cofinite rank and the eulerian cofinite rank of $G$ to correspond to the cases in which $H$ is further restricted to be connected, even, or eulerian, respectively.

Clearly, any graph which has an eulerian chain must be connerter and countable, so this restriction is built into the following synthesis of the conditions of [2] and [3].

THEOREM 1. Let $G$ he a connected countable graph with $n$ odd vertices, cofinite rank $r$ and even cofinite ronk $s$. Then $G$ has an eulorim chain if and only if $n+r \leq$ ? and $s \leq 1$.

We shall in fact prove the following sharper form of this 
characterization.

THEOREM 2. Let $G$ be a connected countable graph with $n$ odd vertices, connected cofinite rank $c$ and eulerian cofinite rank $e$. Then $G$ has an eulerian chain if and only if $n+c \leq 2$ and $e \leq 1$.

In [1] we recently announced, without prof : an intermediate version of these formulations. That version is an immediate corollery of the results in the present paper.

In Section 2 we shall examine some relationships between the various kinds of cofinite rank. The results obtained are applied in Section 3 to deduce Theorems 1 and 2 from the characterizations of Euler and Erdös et al. The paper concludes in Section 4 by briefly examining the independence of the various kinds of cofinite rank.

\section{Cofinite rank relạtionships}

First consider the relationship between cofinite rank and connected cofinite rank.

THEOREM 3. For any connected graph; the cofinite rank and connerted cofinite rank are equal.

Pronf. The theorem is clearly true for finite graphs. Let $G$ be a connected infinite graph with cofinite rank $r$ and connected cofinite rank $c$. By definition, $c \leq r$. We now prove that equality holds. Let $H$ be a disconnected finite subgraph of $G$, and suppose $G \backslash E H$ has $n$ infinite components. Becanse $G$ is connected, there is a peth $P$ in $G \backslash E H$ between two compenents $H_{1}$ and $H_{2}$ of $H$. Let $H^{\prime}:=H \cup P$. Then $H_{1} \cup H_{2} \cup P$ belongs to a single component of $H^{\prime}$, so $H^{\prime}$ has fewer components than $H$. Also $G \backslash E H^{\prime}$ has at least $n$ infinite components. Iteration of this procedure yielis a connected finite subgranh of $G$ whose edge complement has at. least $n$ infinite compnnents, so $n \leq c$. Hence $r \leq c$, and so $r=c$, as renuired.

Next we examine the relationship between even cofinite rank and eulerian cofinite rank.

We shall say that a graph $G$ has property $P$ if, for each finite even subgraph $H$ having exactly two components, the groph G\EH has a 
single infinite component. An important class of quasi-eu?erian graphs has this property.

LEMMA 1. If $G$ is a quasi-eulerion graph with eulerian rofinite renk $I$, then $G$ has property $P$.

Proof. Let $G$ be a quasi-eulerian graph with pulerian cofinite rank 1 . Suppose $G$ does not have property $P$ : we shall show this leads to a contradiction.

By hypothesis, $G$ has an even subgraph $H$, comprising two vertex disjoint eulerian subgraphs $H_{1}$ and $H_{2}$, such that $G \backslash E H$ has at least two infinite components. Since $H$ is finite and $G$ is quasi-eulerian, every component of $G \backslash E H$ is even. If $K$ is any component of $G \backslash E H$, its vertices of attachment are the vertices shared by $K$ and $H$, and its edges of attachment are the edges of $H$ which are incident with the vertices of attachment of $K$. A connecting path for $H$ is a path in $G \backslash E H$ with end vertices in ${ }_{1}$ and $H_{2}$, and no other vertices in common with $H$. Three properties of such paths will now be established.

(1) Every infinite component of $G \backslash E H$ contains a connecting path for $H$.

Let $K$ and $K^{\prime}$ be any two infinite compnnents of $G \backslash E H$, and suppose $K$ does not contain $a$ connerting path for $H$. Then all vertices of sttachment of $K$ must be in one component of $H$, say $H_{I}$. Therefore all edges of attachment of $K$ are in $H_{1}$, so $K$ is a component of $G \backslash E H_{1}$. Also, $G \backslash E H_{1}$ has a component which contains $K^{\prime}$, and $K^{\prime}$ is disjoint, from $\underline{K}$, so $G \backslash E H_{1}$ has at least two infinite components. Because $G$ has eulerian cofinite rank 1 , this contradicts the fact that $H_{1}$ is eulerian. Hence there is no infinite component $K$ of $G \backslash E H$ which dnes not contain a connecting nath for $H$.

(2) No two edge disjoint connecting paths for $H$ have the same end vertices.

Suppose $P$ and $P^{\prime}$ are edge disjoint connecting paths for $H$. Each infinite component of $G \backslash E H$ must contain at least one infinite component of $G \backslash E\left(H \cup P \cup P^{\prime}\right)$, sn this graph has at least two infinite components. 
But $G$ has eulerian cofinite rank 1 sn $H \cup P \cup P^{\prime}$ cannot be eulerian. But $H \cup P \cup P^{\prime}$ is connected, and both components of $H$ are eulerian, so $P$ and $P^{\prime}$ cannot have the same end vertices.

(3) No finite component of $G \backslash E H$ contains a connecting path for H.

Iet $K$ he any finite component of $G \backslash E H$. Since $G$ has eulerian cofinite rank 1 , and the infinite components of $G \backslash E(H \cup K)$ are the same as those of $G \backslash E H$, at least two in number, it fnllows that $H \cup K$ is not eulerian. But $K$ and each component of $H$ are edge disjoint eulerian graphs, so $H \cup K$ is not connected. Therefore $K$ cannot contain a connecting path for $H$.

Let $K_{1}$ and $K_{2}$ be any two infinite components of $G \backslash E H$. Each is incident with $H_{1}$ and $H_{2}$, by property (1). Since $H_{1}$ is eulerian, its edges can be labelled $a_{1}, a_{2}, \ldots, a_{m}$ so that ${ }_{{ }_{1}} a_{1} v_{2} a_{2} \cdots v_{m} a_{1} v_{1}$ is an evlerian circuit with vertices $v_{i}$. Moreover, we may suppose the lahelling to be such that $K_{1}$ is incident with $v_{1}$, and $K_{2}$ is incident with $v_{r}$, while no intervening vertex $v_{i}(1<i<r)$ is incident with any infinite component of $G \backslash E H$. Again, $H_{2}$ is eulerian so its edges can be labelled $b_{1}: b_{2}, \ldots, b_{n}$ so that $w_{1} b_{1} w_{2} b_{2} \cdots w_{n} b_{n} w_{1}$ is an eulerian rirruit with vertices $w_{i}$. We may also suppose the labelling to be such that $w_{1}$ is incident with $K_{1}$, and $w_{s}$ is incident with $K_{2}$, while no intervening vertex $w_{i}(1<i<s)$ is incident with $K_{1}$ or $K_{2}$.

Since $K_{1}$ is connected, it contains a path $P_{1}$ with end vertices " and $w_{1}$. Similarly, there is a path $P_{2}$ in $K_{2}$ with end vertices $v_{p}$ and $w_{s}$. Let $Q_{1}$ and $T_{1}$ be the trails in $H_{1}$ specified by $v_{1} a_{1} v_{2} a_{2} \cdots v_{r}$ and $v_{r} a_{r} \ldots v_{m} a_{m} v_{1}$, respectively. Thus $Q_{1}$ and $T_{1}$ are edge disjoint and $H_{1}=Q_{1} \cup T_{1}$. Similarly = let $Q_{2}$ and $T_{2}$ be the trails in $H_{2}$ specified bv $w_{1} b_{1} w_{2} b_{2} \cdots w_{s}$ and $w_{s} b_{s} \cdots w_{n} b_{n} w_{1}$ respectively. Clearly, $C:=P_{1} \cup T_{1} \cup P_{2} \cup T_{2}$ is a circuit, so an 
eulerian subgraph of $G$. We sha.ll now show that $G \backslash E C$ must have at least two infinite components, contrary to the fact that $G$ has ellerian cofinite rank 1 . This shows that $H$ does not exist, whenre the lemma.

All vertices of attachment of $K_{1}$ and $K_{2}$ are in $T_{1} \cup T_{2}$. Hence the only edges of attachment of $K_{1}$ which are not in $T_{1} \cup T_{2}$ are $a_{1}$ and $b_{1}$. Similarly, the only edges of attachment of $K_{2}$ which are not in $T_{1} \cup T_{2}$ are $a_{p-1}$ and $b_{s-1}$.

Since there are not two edge disjoint paths in $K_{1}$ between $v_{1}$ and $w_{1}$, by property (2), it follows that $v_{1}$ and $w_{1}$ lie in iifferent romponents of $K_{1} \backslash E P_{1}$, which we shall denote by $K_{1}^{\prime}$ and $K_{1}^{\prime \prime}$ respertively. With the possible excention of $v_{1}$, no vertex in $K_{1}^{\prime}$ is odd, because $K_{1}$ is quasi-eulerian; $v_{1}$. mav be ndd or infinite. In either case it follows that $K_{1}^{\prime}$ is infinite. Similarly $K_{1}^{\prime \prime}$ is infinite.

Suppose there is a path $P$ in $G \backslash E C$ between $v_{1}$ and $w_{1}$. Since $K_{1}^{\prime}$ and $K_{1}^{\prime \prime}$ are disjoint, and the only edges of attachment of $K_{1}$ in $G \backslash E\left(T_{1} \cup T_{2}\right)$ are $a_{2}$ and $b_{1}$, so the edges of $P$ incident with $v_{1}$ and $w_{1}$ must be $a_{1}$ and $b_{1}$. This implies that $P$ is edge disjnint. from $K_{1}$. Also, it follows that $P$ contains a connecting path $P^{\prime}$ for with end vertices in $Q_{1}$ and $Q_{2}$. By property (3), $P^{\prime}$ lies in an infinite component of $G \backslash E H$. This must be $K_{2}$, since $P$ is edge disjoint from $K_{1}$, and no other infinite component of $G \backslash E H$ is incident with $Q_{1}$. Hence the end vertices of $P^{\prime}$ are $v_{r}$ and $w_{s}$, so cannot be edge disjoint from $P_{?}$, by property (2), and so $P^{\prime}$ cannot exist in $G \backslash E C$. It follows that there is no path in $G \backslash E C$ between $v_{1}$ and $w_{1}$. Hence $G \backslash E C$ has at least two infinite components, one containing $K_{1}^{\prime}$ and one containing $K_{1}^{\prime \prime}$. This is the required contradiction which shows that $H$ does not exist, and hence that $G$ has property $P$. 
If the graph $G$ has property $P$, there is only one infinite component after the edges of any two disjoint eulerian subgraphs are deleted, so if $H$ is any eulerian subgraph then $G \backslash E H$ has eulerian cofinite rank 1 . This equivalent of property $P$ is useful in proving the next lemma.

LEMMA 2. A quasi-eulerian araph which has eulerian cofinite rank I has even cofinite rank 1 .

Proof. Let $G$ be a quasi-eulerian graph with eulerian cofinite rank 1. Then $G$ has property $\mathrm{P}$, by Lemma 1 . Jet $H$ he a finite even subgraph of $G$ having components $H_{1}, H_{2}, \ldots, H_{n}$. Since $H_{1}$ is eulerian it follows that $G \backslash E H_{1}$ is even, has a single infinite component, and has eulerian cofinite rank 1 . Let. $G_{1}$ be the infinite component of $G \backslash E H_{1}$. Then $G_{1}$ is quasi-eulerian, has ellerian cofinite rank 1 , and so has property $\mathrm{P}$. Also $G \backslash E G_{1}$ is finite and contains $H_{1}$. Clearly $H_{2}, H_{3}, \ldots, H_{n}$ each lie in some comoonent of $G \backslash E H_{1}$. Thus $G_{1} \backslash E H_{2}$ is even, has a single infinite component, and has eulerian cofinite rank 1 . Let $G_{2}$ be the infinite component of $G_{1} \backslash E H_{2}$. Then $G_{2}$ is quasieulerian, has eulerian cofinite rank $I$, and so has property $P$. Also $G \backslash E G_{2}$ is finite and contains $H_{1} \cup H_{2}$. Continuing thus, the quasieulerian graph $G_{n}$ which is iteratively defined by this procedure is such that $G \backslash E G_{n}$ is finite and contains $H$. It follows that one component of $G \backslash E H$ contains $G_{n}$, and any other components are finite. Thus $G \backslash E H$ has a single infinite component.

Since $H$ was arbitrary, $G$ has even cofinite rank 1 .

THEOREM 4. For any quasi-eulerian graph, the eulerian cofinite rank is 1 precisely when the even cofinite rank is 1 .

Pronf. Let $G$ be a quasi-eulerian graph with even cofinite rank $s$ and eulerian cofinite rank $e$, so $e \leq s$. If $s=1$ then $e \leq 1$; but $G$ is infinite, so $e=1$. Conversely, if $e=1$ then $s=1$, by Lemma 2 . 


\section{Characterizing graphs with eulerian chains}

The theorems of Euler and Erdös, Gallai and Vázsonyi characterizing graphs with various kinds of eulerian chain are our essential starting point. They may be stated as follows.

THEOREM 5 (Euler). A connected finite graph has an eulerian circuit precisely if it is even, and has an eulerian trail precisely when it has two odd vertices.

THEOREM 6 (Erdös, Gallai, Vázsonyi). A connected countably infinite graph has an eulerian one-way chain precisely if it has cofinite rank 1 , has at most one odd vertex, and has at least one infinite vertex if it has no odd vertex.

THEOREM 7 (Erdös, Gallai, Vázsonyi). A quasi-eulerian graph has an eulerian two-way chain precisely if it has cofinite rank at most 2 and even cofinite rank 1 .

We now verify that Theorem 1 follows from Theorems 5, 6 and 7 . A graph with exactly one odd vertex must be infinite, so the parameters $n$, $r, s$ in Theorem 1 must have $r \geq 1$ if $n=1$. Also, cofinite rank cannot be less than even cofinite rank, and deletion of a single vertex shows that even cofinite rank is at least 1 for any infinite graph. Thus $s \geq 1$ exactly when $r \geq 1$. Only five triples of parameters meet these conditions and the inequalities of Theorem 1, namely

$$
(n, r, s)=(0,0,0),(2,0,0) .(1,1,1),(0,1,1) \text { and }(0,2,1) \text {. }
$$

The existence of an eulerian chain is guaranteed in the first two cases by Theorem 5, in the next case by Theorem 6, and in the last two cases by Theorem 7. Conversely, for each of the four kinds of eulerian chain, it is trivial to check that the inequalities of Theorem 1 are satisfjed. Thus, Theorem 1 characterizes all graphs with an eulerian chain.

Theorem 2 now follows from Theorem l, using the results established in Theorems 3 and 4.

\section{Independence of the kinds of cofinite rank}

In Theorems 3 and 4 we establisher relations between various kinds of cofinite rank. How far do such relations extend? This question will now be briefly discussed, with regard to connected graphs. (The results for 
disconnerted graphs easilv follow from this discussion.)

Theorem 3 shows that the cofinite and connected cofinite ranks are always equal for a connected graph. However, the even and eulerian cofinite ranks are not always equal. For example, Figure 1 shows a graph which has cofinite rank 2 but, even cofinite rank which is infinite. Indeed. it is not difficult to construct a graph which has any countable number of odd vertices and any eulerian cofinite rank greater than 1 , but for which the even cofinite rank is infinite.

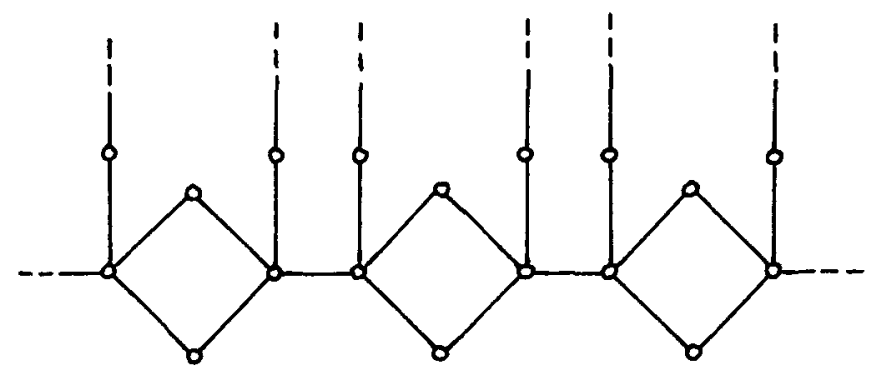

FTGURE 1. A graph with eulerian cofinite rank 2 but even cofinite rank which is infinite.

Theorem 4 shows that, for a quasi-eulerian graph, the eulerian cofinite rank is 1 precisely when the even cofinite rank is 1 . It can readily be shown that this also holds for any connected countable graph with a single odd vertex. On the other hand, Figure 2 shows two graphs which both have more than one odd vertex. and have eulerian cofinite rank 1 but have even cofinite rank greater than 1 . The graphs in Fipure 2 generalize to give a connected, countably infinite graph which has eulerian cofinite rank 1 and any given even cofinite rank. 

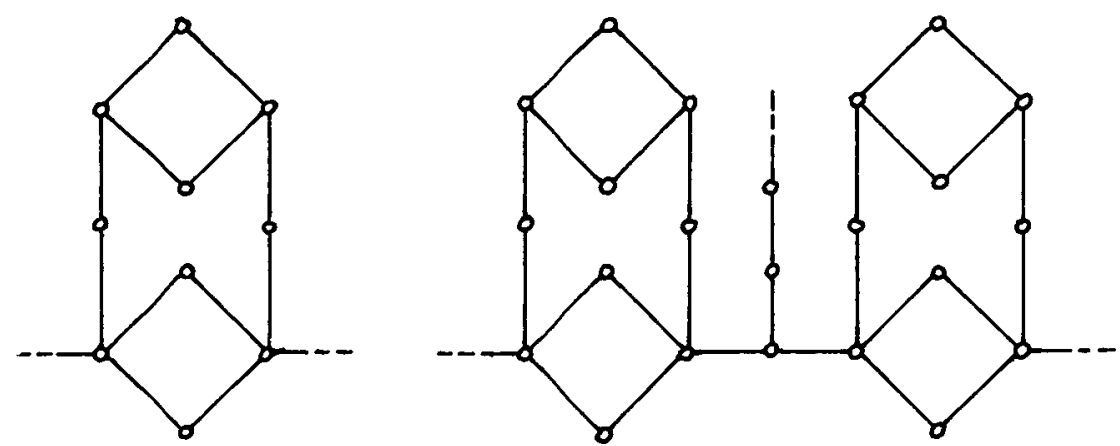

FIGURE 2. Two graphs with eulerian cofinite rank 1; $G$ has even cofinite rank 2 and $\mathrm{H}$ has even cofinite rank 3 .

In conclusion, we note that connected and eulerian cofinite ranks are independent. Figure 3 shows a graph which has eulerian cofinite rank 1 but connected confinite rank which is infinite.

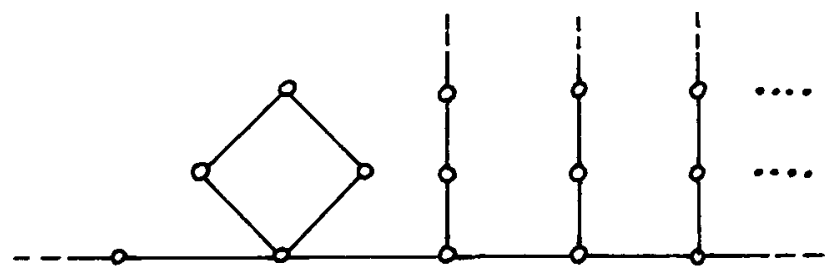

FIGURE 3. A graph with eulerian confinite rank 1 and connected cofinite rank which is infinite.

\section{References}

[1] Roger B. Eggleton and Donald K. Skilton, "Double tracings of graphs", to appear in Proceedings of the 11th Australian Conference on Combinatorial Mathematics, The University of Canterbury, August 29 September 2, 1983.

[2] P. Erdds, T. Grunwald and E. Vázsonyi, "über Euler-Linien unendlicher Graphen", J. Math. Phys. 17 (1983), 59-75. 
[3] L. Euler, "Solutio problematis ad geometriam situs pertinentis", Commentarii Academiae Scientiamm Imperialis Petropolitanae 8 (1736), 128-140. A readily available English translation is given on pp. 3-8 of N. L. Biggs, E. K. Lloyd and R. J. Wilson, Graph Theory 1736-1936, (Oxford University Press 1976).

Department of Mathematics,

The University of Newcastle,

Newcastle,

New South Wales 2308,

Australia. 